\title{
Análise do gerenciamento de resíduos de serviços de saúde de uma instituição de ensino superior em odontologia
}

Os resíduos de serviços de saúde (RSS) necessitam de cuidados especiais devido à presença de agentes químicos e biológicos, que são potencialmente prejudiciais à saúde pública e ambiental. Uma das fontes geradoras de RSS são os serviços odontológicos, cujos resíduos causam risco à saúde pública e ocupacional. É de responsabilidade dos estabelecimentos prestadores de serviços de saúde o gerenciamento dos resíduos por eles gerados através de um Plano de Gerenciamento de Resíduos de Serviços de Saúde (PGRSS). Nesse contexto, o objetivo do trabalho foi analisar o gerenciamento dos RSS e o PGRSS de uma Faculdade de Odontologia vinculada à uma instituição de ensino superior (IES). Os instrumentos de pesquisa utilizados foram o estudo documental do PGRSS da unidade; a observação direta na fonte geradora; e a observação participante. Todos os instrumentos seguiram um roteiro de estudos baseado nas recomendações da legislação brasileira. Os resultados encontrados apontam à existência de falhas tanto no processo de elaboração do PGRSS quanto em sua aplicação na prática das atividades diárias realizadas na unidade geradora. Em vista disso recomenda-se que a gestão de RSS seja parte integrante da formação continuada de professores, técnicos e demais funcionários da faculdade, de forma integrada nas atividades de ensino, pesquisa e extensão, de modo que esse tema seja incluído na rotina da comunidade acadêmica, inclusive como parte da formação acadêmica dos futuros profissionais.

Palavras-chave: Plano de Gerenciamento de Resíduos de Serviços de Saúde; Serviços Odontológicos; Instituição de Ensino Superior.

\section{Healthcare waste management analysis in a higher education institution of dental medicine}

\begin{abstract}
Healthcare waste (HCW) requires special care due mainly to the presence of chemical and biological agents, which are potentially harmful to public and environmental health. One of the generating sources of HCW is dental services, whose waste causes public and occupational health risks. It is the responsibility of health care facilities to manage the waste generated by them through a healthcare waste management plan (HCWMP). In this context, the objective of the study was to evaluate HCW management and the HCWMP of a College of Dental Medicine linked to a higher education institution (HEI). The research instruments used were document analysis of the unit's HCWMP; direct observation of the waste generation source; and participant observation. All research instruments followed a script based on the recommendations of the Brazilian legislation. The results found point to the existence of flaws in the elaboration of the HCWMP and in its application in the practice of daily activities carried out in the facility. Therefore, it is recommended that HCW management be an integral part of teachers, technicians and other faculty members' continuing education, in a way that it is integrated into teaching, research and extension activities, so that this theme is included in the routine of the academic community, even as part of future professionals' academic training.
\end{abstract}

Keywords: Healthcare Waste Management Plan; Dental Services; Higher Education Institution.

Topic: Engenharia Ambiental

Reviewed anonymously in the process of blind peer
Received: 10/08/2018

Approved: 24/09/2018
Micaela Piccoli Terres (D)

Universidade Federal de Pelotas, Brasil http://lattes.cnpq.br/9508122556091933 http://orcid.org/0000-0002-7272-5591 micaelapiccoli@hotmail.com

Mateus Torres Nazari (iD)

Universidade Federal de Pelotas, Brasil http://lattes.cnpq.br/8312954395664621 http://orcid.org/0000-0002-2548-9047 nazari.eas@gmail.com

Carolina da Silva Gonçalves (iD) Universidade Federal de Pelotas, Brasil http://lattes.cnpq.br/8215160183595864 http://orcid.org/0000-0002-2403-4905 carolina.engas@gmail.com

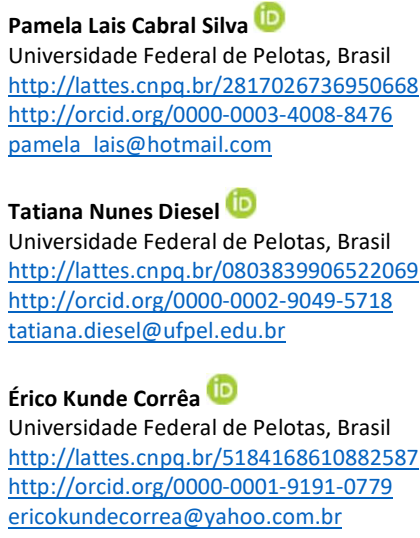

Referencing this:

TERRES, M. P.; NAZARI, M. T.; GONÇALVES, C. S.; SILVA, P. L. C.; DIESEL, T. N.; CORRÊA, É. K.; CORRÊA, L. B.. Análise do gerenciamento de resíduos de serviços de saúde de uma instituição de ensino superior em odontologia. Revista Ibero Americana de Ciências Ambientais, v.9, n.7, p.134-148, 2018. DOI: http://doi.org/10.6008/CBPC2179-6858.2018.007.0013 


\section{INTRODUÇÃO}

Dentre os resíduos produzidos no Brasil, os Resíduos de Serviços de Saúde (RSS) são uma crescente fonte de estudo para profissionais das áreas ambiental e sanitária, devido, principalmente, a presença de agentes químicos e biológicos, considerados prejudiciais à saúde pública e ambiental. A Política Nacional de Resíduos Sólidos (PNRS), instituída pela Lei no 12.305/2010, define gerenciamento como sendo conjunto de ações exercidas, direta ou indiretamente, nas etapas de coleta, transporte, transbordo, tratamento e destinação final ambientalmente adequada dos resíduos sólidos e disposição final ambientalmente adequada dos rejeitos, de acordo com o plano municipal de gestão integrada de resíduos sólidos ou com o plano de gerenciamento de resíduos sólidos (BRASIL, 2010). Desta forma, todo o estabelecimento de saúde deve elaborar e implantar um Plano de Gerenciamento de Resíduos de Serviços de Saúde (PGRSS), buscando a alternativa mais adequada para manejar os resíduos, desde sua fonte geradora até sua correta disposição final, de forma a promover o bem-estar da população e do meio ambiente (TOGNOC, 2015).

No Brasil, os órgãos responsáveis por regular os RSS são a Agência Nacional de Vigilância Sanitária (ANVISA) e o Conselho Nacional do Meio Ambiente (CONAMA). Segundo a Resolução RDC no 306 (BRASIL, 2004) e a Resolução no 358 do CONAMA (BRASIL, 2005), os RSS são classificados em cinco grupos: Grupo A (infectantes), Grupo B (químicos), Grupo C (radioativos), Grupo D (comuns) e Grupo E (perfurocortantes).

Apesar de muitos estabelecimentos possuírem o PGRSS, ainda observam-se problemas em determinadas etapas do manejo de resíduos, assim como o descumprimento do plano na atividade diária. Segundo Corrêa (2005), mesmo com a disponibilidade de legislações e manuais que preconizam condutas de gerenciamento de resíduos nos estabelecimentos prestadores de serviços de saúde, na prática, os quesitos legais são pouco aplicados e os órgãos de fiscalização são pouco atuantes diante da problemática real. Além disso, Hidalgo (2012) relata que a maioria das pesquisas científicas sobre RSS relacionam-se a estudos de quantificação e, consequentemente, poucos pesquisadores têm debatido sobre a gestão desses resíduos em estabelecimentos que prestam serviços odontológicos.

Os RSS de origem odontológica são um dos mais importantes resíduos produzidos em estabelecimentos prestadores de serviços de saúde (BAZRAFSHHAN et al., 2014). O gerenciamento de resíduos odontológicos tem grande relevância social, cientifica e de saúde. Relevância social, pois é importante compreender a repercussão do mau gerenciamento de RSS. Cientifica, visto que se dispõe a alertar as universidades para que promovam suas atividades de ensino, pesquisa e extensão de forma ambientalmente adequada, idealizando um programa de gerenciamento junto à fonte geradora. Na saúde, por entender que um dos mais graves problemas em relação aos resíduos odontológicos deve-se aos danos à saúde causados por acidentes ocupacionais (AMARANTE et al., 2013).

Para Stedile et al. (2015), a odontologia, devido à sua complexidade, é uma fonte especial de geração de RSS, pois, ainda que de pequenas dimensões (consultórios), a diversidade de procedimentos abrange grande variedade de materiais e, consequentemente, de resíduos, assemelhados a certas atividades laboratoriais e de diagnóstico, como raios $\mathrm{X}$, além de caracterizar-se, em microescala, como um centro 
cirúrgico, dependendo das especialidades odontológicas praticadas no estabelecimento. Os mesmos autores consideram que um dos maiores desafios no gerenciamento dos resíduos odontológicos é a localização geográfica, visto que são serviços de pequeno porte, podendo ocupar residências, prédios comerciais, condomínios, e clínicas, associados muitas vezes a diversas atividades comerciais, de serviços e até mesmo industriais, quando a empresa mantém consultório próprio.

Em muitos países subdesenvolvidos os resíduos sólidos provenientes de serviços odontológicos são aterrados juntamente com resíduos domésticos, sem reciclagem ou segregação (NASROLLAHI et al., 2015). Apesar da Resolução no 358 (BRASIL, 2005) e da Resolução RDC nª 306 (BRASIL, 2004) serem claras e específicas quanto ao método de descarte, tratamento e destinação final dos RSS, muitos municípios brasileiros ainda não possuem um plano de gerenciamento para essa classe de resíduos implementado adequadamente, como é demonstrado frequentemente através dos meios de comunicação que relatam flagrantes de descarte de RSS em lixões a céu aberto (ALVES et al., 2016). Esses autores apontam que os principais fatores motivadores para o descarte inadequado de RSS são a imprudência, a carência de conhecimento sobre a importância de um adequado gerenciamento e a impunidade.

As instituições de ensino superior (IES), sendo responsáveis pela produção e socialização do conhecimento e formação de recursos humanos, têm o papel de dar o exemplo (produzir, socializar e formar respeitando o meio ambiente) e atualizar e monitorar a execução do PGRSS, possibilitando seu aprimoramento contínuo (STEDILE et al., 2015). Contudo, para Oliveira et al. (2012), cirurgiões-dentistas ainda não possuem conhecimento e atitudes suficientes e satisfatórias quanto ao gerenciamento dos resíduos produzidos em seus estabelecimentos e, apesar de sua obrigatoriedade, muitos profissionais desconhecem ou não possuem um PGRSS nos locais de trabalho. O mesmo estudo destaca ainda a importância em expor conhecimentos de biossegurança voltados ao descarte de resíduos nos cursos de graduação, visto que a não inclusão desses conhecimentos no processo de formação de futuros profissionais pode justificar o que acontece atualmente com os RSS, tanto intra como extra estabelecimentos de saúde.

O presente trabalho tem sua fonte geradora inserida em uma Universidade, o que, diferentemente do estudo em clínicas odontológicas, demonstra a possibilidade de integrar a educação ambiental na formação de futuros profissionais, tornando-os instrumentalizados para gerenciarem os RSS de forma adequada no seu ambiente de trabalho. No Brasil, há um total de 219 Faculdades de Odontologia. No entanto, há carência na produção de conhecimento sobre RSS dessa fonte geradora, acentuando a importância do aprofundamento de estudos que investiguem a situação das instituições de formação, especialmente públicas, no seu processo de adaptação ao formato de gerenciamento introduzido pela PNRS. Diante disso, o objetivo deste estudo foi analisar o gerenciamento dos RSS e o PGRSS de uma faculdade de odontologia vinculada à uma instituição de ensino superior (IES).

\section{METODOLOGIA}

Este trabalho foi realizado no prédio da Faculdade de Odontologia de uma instituição de ensino superior, localizada na região sul do Brasil. Nesse local são desenvolvidas atividades teóricas, em salas de 
aula, e práticas, em laboratórios e clínicas distribuídos em sete andares, numa área total de $6.528 \mathrm{~m}^{2}$. Atualmente, a Faculdade de Odontologia conta com 66 professores, 46 técnicos administrativos e 568 acadêmicos de graduação, 103 acadêmicos de pós-graduação stricto sensu e 13 acadêmicos de pósgraduação lato sensu.

Os serviços odontológicos oferecidos pela Faculdade de Odontologia são: atendimentos de Dentística, Periodontia, Cirurgia, Endodontia, Odontopediatria, Estomatologia e Prótese Dentária e Radiologia. Com 107 cadeiras odontológicas disponíveis, a capacidade de atendimento é de 320 pacientes ao mês. As atividades clínicas desenvolvidas pelos professores, técnicos administrativos e acadêmicos do curso de odontologia compreendem a prestação de atendimento odontológico à população.

Este trabalho consiste em um estudo exploratório e descritivo da situação do gerenciamento dos RSS na Faculdade de Odontologia, após a implantação do seu PGRSS. A pesquisa exploratória tem como objetivo proporcionar maior familiaridade com o problema em estudo, com vistas a torná-lo mais explícito ou a construir hipóteses (SILVEIRA et al., 2009). A pesquisa descritiva exige do pesquisador uma série de informações sobre o que se deseja pesquisar, uma vez que esse tipo de estudo busca descrever os fatos e fenômenos de determinada realidade documental (SILVEIRA et al., 2009).

Para este trabalho, foram empregados três instrumentos de coleta de dados nas visitas in loco realizadas: a) observação direta na fonte geradora; b) observação participante; c) análise documental. Para todos os instrumentos de pesquisa foi realizado um roteiro de estudos baseado nas etapas recomendadas pelo Manual de Gerenciamento de Resíduos de Serviços de Saúde da Anvisa (BRASIL, 2006), sendo elas: 1) Identificação do problema; 2) Definição da equipe de trabalho; 3) Mobilização da organização; 4) Diagnóstico da situação dos RSS; 5) Definição de metas, objetivos, período de implantação e ações básicas; 6) Elaboração do PGRSS; 7) Implementação do PGRSS; e 8) Avaliação do PGRSS.

Segundo Moreira (2002, citado por OLIVEIRA, 2008), a observação participante é conceituada como sendo "uma estratégia de campo que combina ao mesmo tempo a participação ativa com os sujeitos, a observação intensiva em ambientes naturais, entrevistas abertas informais e análise documental". Já a observação direta consiste no contato pessoal do pesquisador com o objeto estudado, possibilitando que ele utilize seus conhecimentos e experiências como auxiliares na compreensão e interpretação desse objeto (MAXIMIANO et al., 2006). Por fim, a pesquisa documental é muito próxima da pesquisa bibliográfica, diferenciando-se apenas na natureza das fontes, pois enquanto a pesquisa bibliográfica remete para as contribuições de diferentes autores sobre o tema (fontes secundárias), a pesquisa documental recorre a materiais que ainda não receberam tratamento analítico (fontes primárias) (SÁ-SILVA et al., 2009).

As informações obtidas foram divididas de acordo com as etapas descritas em um roteiro de estudos, para a melhor visualização dos resultados. A discussão dos resultados foi realizada através da análise documental do PGRSS da Faculdade de Odontologia e da observação crítica e sistêmica das condições atuais de gerenciamento dos RSS na instituição. Para tanto, utilizou-se o Manual de Gerenciamento de Resíduos de Serviços de Saúde da Anvisa (BRASIL, 2006), a Resolução RDC na 306 (BRASIL, 2004) e a Resolução CONAMA no 358 (BRASIL, 2005) como suporte para a identificação de não conformidades com a legislação. 


\section{RESULTADOS E DISCUSSÃO}

Não há dúvidas de que, se seguidas as legislações ambientais referentes aos RSS, os efeitos negativos causados por este tipo de resíduo podem ser reduzidos ou até mesmo eliminados, sendo que a minimização destes efeitos negativos está fortemente relacionada com o comportamento dos profissionais da área da saúde nos estabelecimentos de saúde (DARWISH et al., 2006). Muitos países desenvolvidos, como os Estados Unidos, já tem um sistema completo de gerenciamento de RSS estabelecido (BOS et al., 2002), porém, os países subdesenvolvidos ainda sofrem com a disposição incorreta de resíduos, falta de recursos financeiros, carência de conhecimento sobre a periculosidade que os RSS apresentam à saúde humana e poucas informações sobre geração e disposição final deste tipo de resíduo (DARWISH et al., 2006).

Posto que esses tipos de estabelecimentos são responsáveis pelo correto gerenciamento de todos os RSS por eles gerados (BRASIL, 2006), surge a demanda para a elaboração e implementação de um PGRSS na Faculdade de Odontologia da Universidade Federal de Pelotas, visando a obtenção de licenciamento junto ao órgão ambiental responsável. Apesar da preexistência de um plano de gerenciamento de resíduos na unidade, este se encontrava desatualizado e não seguia as recomendações legais vigentes. Esses dois fatores impulsionaram a mobilização da direção para a criação de um PGRSS que considerasse tanto as particularidades de uma IES como fonte geradora de RSS, quanto às exigências da legislação para o correto gerenciamento dos resíduos (Tabela 1).

Tabela 1: Verificação das ações realizadas da etapa 1 no PGRSS da Faculdade de Odontologia (FO).

\begin{tabular}{|c|c|}
\hline Etapas para elaboração do PGRSS (BRASIL, 2006) & Análise documental do PGRSS da FO \\
\hline \multicolumn{2}{|l|}{ Identificação do problema } \\
\hline - Obter o apoio da direção da instituição & $\checkmark$ \\
\hline $\begin{array}{l}\text { - Reconhecimento do problema e sinalização positiva da } \\
\text { administração para início do processo }\end{array}$ & $\sqrt{ }$ \\
\hline
\end{tabular}

Legenda: $\checkmark$ (realizado); $X$ (não realizado).

De acordo com Moura (2004), promover a mudança de hábitos em um estabelecimento já em funcionamento é mais difícil, uma vez que há uma tendência natural de cada setor em achar que não é o responsável pelo problema, resistindo às mudanças, pois "sempre foi feito assim". Nesse contexto, o autor afirma que, para as instituições interessadas em implantar um plano de gerenciamento é imprescindível que haja um apoio muito forte da alta direção, sem o qual o plano está fadado ao fracasso.

Em concordância com as exigências legais (BRASIL, 2006), a Comissão Gestora para o PGRSS foi constituída pela direção da Faculdade de Odontologia, objetivando a elaboração e implantação do PGRSS na instituição. Esta comissão presta assessoria técnico-científica em caráter permanente e inclui profissionais capacitados das áreas ambiental e odontológica, cumprindo a segunda etapa para elaboração do PGRSS (Tabela 2).

Devido à complexidade associada às características dos RSS, torna-se necessário a inclusão de profissionais com formação ao mesmo tempo específica e abrangente, assim como equipes multidisciplinares que consigam estudar a situação da forma mais ampla possível, visando alcançar a máxima eficiência no gerenciamento e evitar os riscos à saúde ambiental em seus diversos aspectos (SCHNEIDER et al., 2015). 
Tabela 2: Verificação das ações realizadas da etapa 2 no PGRSS da Faculdade de Odontologia (FO).

\begin{tabular}{|l|l|}
\hline Etapas para elaboração do PGRSS (BRASIL, 2006) & \multicolumn{1}{|l|}{ Análise documental do PGRSS da FO } \\
\hline Definição da equipe de trabalho \\
\hline $\begin{array}{l}\text { - Designar profissional para a elaboração e implantação do } \\
\text { PGRSS e compor equipe de trabalho }\end{array}$ & $\checkmark$ \\
\hline
\end{tabular}

Legenda: $\checkmark$ (realizado); $X$ (não realizado).

A etapa de mobilização da organização abrange a sensibilização dos envolvidos para a realização do PGRSS, resultando no conhecimento e envolvimento de todos os funcionários na importância de se gerenciar os RSS e na compreensão, execução, implantação e manutenção do PGRSS (BRASIL, 2006). A Tabela 3 apresenta alguns passos recomendados para a realização dessa etapa e seu status de execução no PGRSS estudado neste trabalho.

Tabela 3: Verificação das ações realizadas da etapa 3 no PGRSS da Faculdade de Odontologia (FO).

\begin{tabular}{|l|l|}
\hline Etapas para elaboração do PGRSS (BRASIL, 2006) & $\begin{array}{l}\text { Análise documental do } \\
\text { PGRSS da FO }\end{array}$ \\
\hline Mobilização da organização & $\checkmark$ \\
\hline $\begin{array}{l}\text { - Promover reuniões com os vários setores para apresentar a ideia, o possível esquema de trabalho } \\
\text { e o que é esperado de cada unidade } \\
\text { - Promover atividades de sensibilização sobre a importância do PGRSS }\end{array}$ & \\
\hline $\begin{array}{l}\text { - Preparar um questionário para levantar a percepção dos funcionários sobre o meio ambiente, de } \\
\text { forma a identificar eventuais questões chaves relacionadas aos RSS }\end{array}$ & $X$ \\
\hline
\end{tabular}

Legenda: $\checkmark$ (realizado); $X$ (não realizado).

Em relação à sensibilização e capacitação dos profissionais envolvidos no processo de gestão de resíduos, a Faculdade de Odontologia se compromete, em seu PGRSS, em realizar encontros com os profissionais da prestação. Neste contexto, desde a implementação do plano, no início de 2016, foram realizados dois encontros, nos meses de março e junho, organizados pela direção da Faculdade de Odontologia, conforme consta em seu PGRSS. O primeiro encontro foi realizado com os técnicos administrativos da Faculdade de Odontologia, a fim de esclarecer e reforçar a importância do uso dos Equipamentos de Proteção Individual (EPIs), bem como da adoção de rotinas na clínica odontológica, buscando evitar acidentes com perfurocortantes, e a importância da correta segregação de resíduos.

A empresa terceirizada responsável pela execução dos serviços de limpeza e conservação dos ambientes clínicos e não clínicos da Faculdade de Odontologia não fornece uma capacitação específica para o manejo de RSS aos seus funcionários. Portanto, o segundo encontro, realizado em junho de 2016, buscou promover a sensibilização na gestão de resíduos e foi direcionado aos profissionais da higienização, objetivando garantir a segurança e a qualidade das atividades desenvolvidas. Schneider et al. (2015) destacam a importância da aplicação de questionários como um indicativo do nível de conhecimento dos profissionais com relação à questão dos RSS. No que se refere ao presente estudo, não houve aplicação de questionários prévios sobre o conhecimento dos funcionários e servidores da Faculdade de Odontologia sobre os RSS e seu gerenciamento em virtude da urgência pela elaboração do PGRSS. Logo, é recomendado que esse questionamento seja feito durante o período de implementação do plano de gerenciamento, podendo ser usado como um instrumento de avaliação e indicação de possíveis pontos de melhoria.

O estudo da situação do estabelecimento prestador de serviços de saúde em relação aos RSS identifica suas condições e áreas críticas, fornecendo os dados necessários para a implantação do plano de 
gestão (BRASIL, 2006). O diagnóstico da situação do gerenciamento de RSS na Faculdade de Odontologia não foi realizado, conforme indica a Tabela 4.

Tabela 4: Verificação das ações realizadas da etapa 4 no PGRSS da Faculdade de Odontologia (FO).

\begin{tabular}{|c|c|}
\hline Etapas para elaboração do PGRSS (BRASIL, 2006) & Análise documental do PGRSS da FO \\
\hline Diagnóstico da situação dos RSS & \\
\hline $\begin{array}{l}\text { - Elaborar um relatório baseado em fatos comprobatórios e na } \\
\text { pesquisa realizada abordando as seguintes questões: } \\
\text { a) a descrição de todos os procedimentos relacionados à } \\
\text { gestão dos RSS (identificação dos resíduos gerados, } \\
\text { acondicionamento, coleta e transporte interno, fluxo de coleta } \\
\text { interno, quantificação dos RSS, armazenamento interno e externo, } \\
\text { área de higienização, coleta e transporte externo, tratamento, } \\
\text { disposição final, política de gestão ambiental e capacitação); } \\
\text { b) os aspectos problemáticos; } \\
\text { c) as referências às legislações, regulamentos, normas etc. }\end{array}$ & $\mathrm{X}$ \\
\hline
\end{tabular}
Legenda: $\checkmark$ (realizado); $X$ (não realizado).

Schneider et al. (2015) citam a realização de um diagnóstico abrangente e detalhado da forma de manejo dos resíduos, que revele o cenário atual do estabelecimento, como uma etapa fundamental para o sucesso do PGRSS. O Manual de Gerenciamento de Resíduos (SISTEMA FIRJAN, 2006) também confirma a importância dessa etapa para o plano de gerenciamento e seu contínuo monitoramento observando que "não se pode gerenciar o que não se conhece". Considerando-se a relevância do diagnóstico para o sucesso da gestão de resíduos, sua realização é indicada, mesmo com a conclusão do PGRSS, para avaliação da aplicabilidade e efetividade do plano de gerenciamento elaborado para a Faculdade de Odontologia.

O PGRSS da Faculdade de Odontologia objetiva realizar o gerenciamento dos RSS a partir da minimização da geração e do correto destino final dos resíduos, buscando a redução dos impactos negativos referentes às atividades por eles realizadas, e possibilitar a saúde e o bem-estar humano e ambiental, conforme a legislação vigente. Ainda que definidos os objetivos para o gerenciamento dos RSS dessa Faculdade, o PGRSS não propõe um cronograma de ações básicas e prazos relativos ao seu cumprimento (Tabela 5).

Tabela 5: Verificação das ações realizadas da etapa 5 no PGRSS da Faculdade de Odontologia (FO).

\begin{tabular}{|l|l|}
\hline Etapas para elaboração do PGRSS (BRASIL, 2006) & Análise documental do PGRSS da FO \\
\hline Definição de metas, objetivos, período de implantação e ações básicas \\
\hline $\begin{array}{l}\text { - Decidir quais as metas a serem atingidas, definir cronograma e } \\
\text { construir os objetivos que levarão ao atingimento das metas }\end{array}$ & $X$ \\
\hline $\begin{array}{l}\text { - Dimensionar a equipe de trabalho, relacionando número de } \\
\text { empregados, cargos, formação e responsabilidade técnica }\end{array}$ & $\checkmark$ \\
\hline $\begin{array}{l}\text { - Relacionar e quantificar os investimentos necessários para a } \\
\text { implantação e avaliação do PGRSS }\end{array}$ & $X$ \\
\hline
\end{tabular}
Legenda: $\checkmark$ (realizado); $X$ (não realizado).

Segundo Schneider et al. (2015), a utilização de ferramentas de gerenciamento na elaboração do PGRSS auxilia no estabelecimento de metas e no cronograma de execução das atividades, ao passo que contribui para esclarecer quais são as ações prioritárias referentes ao manejo de resíduos nos estabelecimentos de saúde. De acordo com Lobato et al. (2009, citado por SCHNEIDER et al., 2015), ter um plano de ação contribui para alcançar os objetivos propostos, bem como à implementação de uma solução para inesperados problemas e prioridades. Nesse caso, foi recomendado o emprego da ferramenta $5 \mathrm{~W} 2 \mathrm{H}$ 
(do inglês: Why - por que, What - o que, Where - onde, When - quando, Who-quem, How - como e How much - quanto custa), bastante útil à hierarquização das prioridades, de cunho essencialmente gerencial, buscando o simples entendimento através da definição de responsabilidades, métodos, prazos, objetivos e recursos necessários, resultando na definição clara de um plano de ação (SCHNEIDER et al., 2015).

No PGRSS da Faculdade de Odontologia não foram relacionados e quantificados os investimentos necessários para sua implantação e avaliação (Tabela 5). Todavia, Schneider et al. (2015) ressalta a importância de estarem previstos e descritos no documento os custos relacionados ao sistema de gerenciamento. A autora ainda acredita que essa etapa é de extrema importância, especialmente por se tratar de recursos monetários provenientes do Governo Federal.

Embora compulsória, a elaboração do PGRSS deve ser encarada como uma ação essencial ao aprimoramento da gestão de resíduos nos serviços de saúde, pois possibilita a compilação de informações e conduz à prática reflexiva, fundamental à promoção da mudança, na forma como esses resíduos vêm sendo manejados nas instituições geradoras (SCHNEIDER et al., 2015). As etapas de elaboração e implementação do PGRSS foram englobadas num mesmo tópico, visto que ocorreram de forma simultânea na Faculdade de Odontologia. As observações referentes à estas etapas foram divididas nas Tabelas 6, 7 e 8.

Tabela 6: Verificação das ações realizadas da etapa 6 no PGRSS da Faculdade de Odontologia (FO).

\begin{tabular}{|c|c|}
\hline Etapas para elaboração do PGRSS (BRASIL, 2006) & Análise documental do PGRSS da FO \\
\hline \multicolumn{2}{|l|}{ Elaboração do PGRSS } \\
\hline \multicolumn{2}{|l|}{ Dados sobre o estabelecimento } \\
\hline $\begin{array}{l}\text { - Informar os dados gerais do estabelecimento } \\
\text { - Informar os componentes da equipe que elabora e implementa } \\
\text { o PGRSS, com identificação da ART } \\
\text { - Informar a caracterização do estabelecimento } \\
\text { - Informar quais são as atividades e serviços predominantes }\end{array}$ & $\checkmark$ \\
\hline \multicolumn{2}{|l|}{ Abastecimento de água / Efluentes líquidos } \\
\hline $\begin{array}{l}\text { - Informar qual o sistema de abastecimento } \\
\text { - Informar se existe aplicação de produtos químicos na água para } \\
\text { o abastecimento } \\
\text { - Informar se existe o controle interno ou externo de qualidade } \\
\text { da água } \\
\text { - Informar a forma de esgotamento sanitário dos efluentes } \\
\text { - Informar se existe tratamento ou não dos efluentes no } \\
\text { estabelecimento ou na rede coletora }\end{array}$ & $\mathrm{X}$ \\
\hline \multicolumn{2}{|l|}{ Emissões gasosas } \\
\hline $\begin{array}{l}\text { - Informar se existe geração de vapores e gases, identificar e } \\
\text { localizar os pontos de geração }\end{array}$ & $\checkmark$ \\
\hline \multicolumn{2}{|l|}{ Tipos e quantidades de resíduos gerados } \\
\hline $\begin{array}{l}\text { - Identificar e quantificar os tipos de resíduos gerados no } \\
\text { estabelecimento em cada setor gerador }\end{array}$ & $\checkmark$ \\
\hline
\end{tabular}

Legenda: $\checkmark$ (realizado); $X$ (não realizado).

No PGRSS da Faculdade de Odontologia consta um Memorial Descritivo de todas as atividades desenvolvidas na unidade de acordo com sua localização, por andar do prédio. Além disso, o plano apresenta a caracterização do estabelecimento e designa um Responsável Técnico pelo PGRSS. O PGRSS apresenta os locais de emissão/geração de gases/resíduos e identifica os resíduos gerados, por setor gerador. O volume de RSS gerado é registrado separadamente, em uma planilha de dados. No entanto, não é feita a medição da geração de RSS por grupo de resíduos, sendo quantificado apenas o volume, em litros, do total de RSS gerado semanalmente. 
Conforme definido na Resolução RDC no 306 (BRASIL, 2004) e na Resolução CONAMA no 358 (BRASIL, 2005), os resíduos gerados devem ser analisados por categorias, sendo que, dentre os resíduos do Grupo $D$ pode-se também avaliar o potencial de reciclabilidade (SCHNEIDER et al., 2015). Conhecer as taxas de geração de RSS através de caracterização possibilita alterações nos processos de trabalho e manejo de resíduos, assim como o estabelecimento de medidas para aperfeiçoar sua eficácia (SCHNEIDER et al., 2015).

Tabela 7: Verificação das ações realizadas da etapa 6 no PGRSS da Faculdade de Odontologia (FO).

\begin{tabular}{|c|c|c|}
\hline Etapas para elaboração do PGRSS (BRASIL, 2006) & $\begin{array}{l}\text { Análise } \\
\text { documental do } \\
\text { PGRSS da FO }\end{array}$ & $\begin{array}{l}\text { Inserção do } \\
\text { PGRSS na FO }\end{array}$ \\
\hline \multicolumn{3}{|l|}{ Elaboração do PGRSS } \\
\hline \multicolumn{3}{|l|}{ Segregação } \\
\hline $\begin{array}{l}\text { - Informar as formas de segregação que serão adotadas para os grupos A, B, C, D (incluindo } \\
\text { os recicláveis) e; }\end{array}$ & $\checkmark$ & $\mathrm{X}$ \\
\hline - Informar quais os EPIs e EPCs utilizados & $\checkmark$ & $\checkmark$ \\
\hline \multicolumn{3}{|l|}{ Tipo de acondicionamento } \\
\hline $\begin{array}{l}\text { - Descrever como e onde serão acondicionados os resíduos dos grupos A, B, D e, } \\
\text { considerando os tipos de contenedores, sacos plásticos, bombonas, salas de resíduos, } \\
\text { abrigo e suas identificações em função do tipo de resíduos nas áreas internas e externas do } \\
\text { estabelecimento }\end{array}$ & $\checkmark$ & $\checkmark$ \\
\hline \multicolumn{3}{|l|}{ Coleta interna } \\
\hline $\begin{array}{l}\text { - Descrever as formas de coleta, tipos de recipientes, carros de coleta, equipe, frequência } \\
\text { e roteiros adotados }\end{array}$ & $\checkmark$ & $\checkmark$ \\
\hline $\begin{array}{l}\text { - Determinar a rotina e frequência de coleta para cada unidade ou setor do } \\
\text { estabelecimento }\end{array}$ & $\checkmark$ & $\checkmark$ \\
\hline \multicolumn{3}{|l|}{ Transporte interno } \\
\hline $\begin{array}{l}\text { - Informar como serão os transportes internos de resíduos, se separadamente, em carros, } \\
\text { ou recipientes coletores específicos a cada grupo de resíduos }\end{array}$ & $\checkmark$ & $\checkmark$ \\
\hline $\begin{array}{l}\text { - Definir os tipos e quantidade de carros coletores que serão utilizados para o transporte } \\
\text { de cada grupo de resíduos, capacidade dos carros, identificação e cores }\end{array}$ & $\checkmark$ & $\checkmark$ \\
\hline \multicolumn{3}{|l|}{ Armazenamento para coleta externa dos RSS } \\
\hline $\begin{array}{l}\text { - Informar a quantidade de contenedores a ser utilizada para cada grupo de RSS, } \\
\text { capacidade volumétrica de cada um e disposição na área }\end{array}$ & $\checkmark$ & $\checkmark$ \\
\hline $\begin{array}{l}\text { - Informar como são higienizados o abrigo, os contenedores, carros coletores e com que } \\
\text { frequência }\end{array}$ & $\mathrm{X}$ & $\checkmark$ \\
\hline \multicolumn{3}{|l|}{ Coleta e transporte externo dos RSS } \\
\hline - Informar quem realiza a coleta externa & $\checkmark$ & $\checkmark$ \\
\hline $\begin{array}{l}\text { - Informar o tipo de veículo utilizado para o transporte e a rotina e frequência de coleta } \\
\text { externa do estabelecimento para os diferentes tipos de resíduos gerados }\end{array}$ & $\checkmark$ & $\checkmark$ \\
\hline $\begin{array}{l}\text { - Informar o destino dos resíduos coletados, por tipo, e anexar os documentos } \\
\text { comprobatórios das empresas coletoras }\end{array}$ & $\checkmark$ & $\checkmark$ \\
\hline \multicolumn{3}{|l|}{ Tratamento dos RSS } \\
\hline - Descrever o tratamento interno para os resíduos, especificados por tipo de resíduo & $\checkmark$ & $\checkmark$ \\
\hline $\begin{array}{l}\text { - Descrever os tipos de tratamento externo adotados para cada grupo de resíduos e quais } \\
\text { os equipamentos e instalações de apoio, incluindo os seguintes aspectos: } \\
\text { a) tecnologias de tratamento adotadas; } \\
\text { b) nome da empresa responsável; } \\
\text { c) localização das unidades de tratamento; } \\
\text { d) responsável técnico pelo sistema de tratamento. }\end{array}$ & $\checkmark$ & $\checkmark$ \\
\hline - Anexar os documentos comprobatórios dos sistemas e tecnologias adotados & $\checkmark$ & $\checkmark$ \\
\hline \multicolumn{3}{|l|}{ Disposição final } \\
\hline - Informar as formas de disposição final dos RSS e especificar por tipo de resíduos & $\checkmark$ & $\checkmark$ \\
\hline $\begin{array}{l}\text { - Informar quais as empresas que executam a disposição final dos RSS e anexar os } \\
\text { documentos comprobatórios de que a empresa está apta a realizar o serviço }\end{array}$ & $\checkmark$ & $\checkmark$ \\
\hline
\end{tabular}

Legenda: $\checkmark$ (realizado); $X$ (não realizado).

As etapas de segregação e coleta interna da Faculdade de Odontologia apresentam algumas falhas de execução. Recipientes para segregação e acondicionamento dos resíduos domiciliares (Grupo D), identificados nas cores verde (recicláveis) e laranja (comuns), como previsto no PGRSS, podem ser 
encontrados nos corredores de todos os andares do prédio onde está localizada a Faculdade de Odontologia. Apesar da presença destes recipientes e da colaboração daqueles que segregam esta classe de resíduo corretamente, os resíduos recicláveis e comuns, acondicionados em sacos plásticos de cor preta, são misturados no momento da coleta interna. Consequentemente, os resíduos recicláveis, que poderiam ser direcionados para o Programa de Coleta Seletiva do município, são coletados via Coleta Domiciliar, junto aos resíduos orgânicos.

Outro problema marcante na segregação de resíduos refere-se ao resíduo gerado no processo de esterilização dos instrumentos odontológicos utilizados pelos acadêmicos para atendimentos nas clínicas. Os instrumentos odontológicos que tiverem contato com resíduos infectantes são autoclavados em embalagens de papel grau cirúrgico não reutilizáveis. O resíduo (embalagem de papel grau cirúrgico) gerado nesse processo é reciclável e não infectante (Grupo A). Porém, durante observação direta na fonte geradora, constatou-se o descarte incorreto desse material junto ao resíduo A, que é acondicionado em sacos branco leitosos identificados pelo símbolo de substância infectante. Os resíduos perfurocortantes (Grupo E) são acondicionados em caixas coletoras descarpack, distribuídas nas clínicas da Faculdade de Odontologia.

Considerando a capacidade de atendimento ao público e o número de acadêmicos da Faculdade de Odontologia, especula-se que haja geração significativa do resíduo de embalagem de papel grau cirúrgico e que ele seja, em sua grande maioria, descartado junto aos resíduos contaminados. Essa especulação é baseada em informações obtidas através de observação participante, onde um aumento no volume da geração de resíduos do Grupo B foi observado, ao mesmo tempo em que o papel grau cirúrgico foi adotado como método de esterilização, há cerca de dezoito meses. No método de esterilização antigo, a embalagem empregada era reutilizável. Contudo, não há, no momento, como comprovar se este é o real motivo do aumento de resíduos do Grupo B, já que não foi realizada a caracterização e análise quali-quantitativa da geração destes resíduos.

Hidalgo (2012) ressalta que quando os resíduos comuns são dispostos junto aos resíduos contaminados, também acabam por se contaminar. O custo para tratamento e disposição final adequada dos resíduos do Grupo A representa um gasto significativo à instituição, sendo necessário priorizar a minimização da geração destes resíduos, promovendo benefícios não só ambientais, mas também econômicos, evitando o uso desnecessário de recursos financeiros públicos (HIDALGO, 2012). Para tanto, a autora sugere que, durante as aulas práticas, onde os alunos prestam atendimento odontológico ao público, a segregação correta dos resíduos gerados, especialmente a embalagem de papel grau cirúrgico, também seja avaliada e pontuada, como é a execução dos procedimentos médicos.

As etapas de classificação e segregação dos RSS são a base de um sistema de gerenciamento e, quando eficientes, levam a melhores desempenhos quanto aos custos, gerando benefícios particulares e difusos (SCHNEIDER, 2015). A autora ainda considera que a gestão de resíduos é um mecanismo eficaz, tanto sob o aspecto socioambiental quanto sob o aspecto econômico, fazendo com que a combinação destes fatores, na forma do triple bottom line, ou tripé da sustentabilidade (People - pessoas, parcela social; Planet - planeta, parcela ambiental; e Profit - lucro, parcela econômica), forme o conceito de sustentabilidade, 
transcendendo uma concepção simplesmente teórica e assumindo relevância prática nas atividades modernas.

A coleta interna dos resíduos produzidos nas clínicas da Faculdade de Odontologia é realizada diariamente, nos horários de menor fluxo de pacientes e acadêmicos da unidade: início da manhã, início da tarde e final da tarde. O transporte interno dos resíduos até seu local de armazenamento externo, é feito pelo elevador aos fundos da unidade, com o auxílio de carros de coleta. Apesar de prevista no PGRSS, não é informado o método ou frequência de higienização do local de armazenamento externo. Foi observado que o portão de acesso ao local de armazenamento externo permanece aberto durante o dia, permitindo a entrada de pessoal não autorizado, bem como seu livre acesso aos resíduos ali armazenados.

As etapas de coleta externa, tratamento e disposição final dos resíduos dos Grupos A, B e E são realizadas por empresas especializadas, devidamente licenciadas pelo órgão ambiental responsável, conforme o PGRSS da Faculdade de Odontologia. A coleta externa é realizada uma vez na semana e acompanhada por um membro técnico. Essa etapa é realizada por dois funcionários devidamente uniformizados, identificados e com o uso dos EPIs necessários. O transporte externo das treze bombonas de 200 L, usadas para o armazenamento externo dos resíduos perigosos, é realizado em conformidade com as exigências para o transporte rodoviário de produtos e/ou resíduos perigosos, segundo Licença de Operação emitida pelo órgão ambiental responsável. A disponibilização e higienização das bombonas é realizada pela mesma empresa responsável pelo tratamento dos RSS.

Tabela 8: Verificação das ações realizadas da etapa 6 no PGRSS da Faculdade de Odontologia (FO).

\begin{tabular}{|c|c|}
\hline Etapas para elaboração do PGRSS (BRASIL, 2006) & $\begin{array}{l}\text { Análise documental do PGRSS da } \\
\text { Faculdade de Odontologia }\end{array}$ \\
\hline \multicolumn{2}{|l|}{ Elaboração do PGRSS } \\
\hline \multicolumn{2}{|l|}{ Situações de emergência e acidentes } \\
\hline - Descrever as ações a serem adotadas em situações de acidentes & $\mathrm{X}$ \\
\hline \multicolumn{2}{|l|}{ Capacitação } \\
\hline $\begin{array}{l}\text { - Descrever as capacitações a serem realizadas, nas formas inicial e educação } \\
\text { continuada }\end{array}$ & $x$ \\
\hline \multicolumn{2}{|l|}{ Indicadores de execução e avaliação } \\
\hline $\begin{array}{l}\text { - Informar quais os indicadores utilizados para o acompanhamento da } \\
\text { execução/implementação do PGRSS e a medição de seu impacto }\end{array}$ & $\checkmark$ \\
\hline \multicolumn{2}{|l|}{ Implementação do PGRSS } \\
\hline $\begin{array}{l}\text { - Estabelecer, das ações, procedimentos e rotinas concebidos no PGRSS, os prioritários, } \\
\text { indispensáveis ao início da operação }\end{array}$ & $\checkmark$ \\
\hline - Fazer o acompanhamento estratégico e operacional das ações & \\
\hline
\end{tabular}

Legenda: $\checkmark$ (realizado); $X$ (não realizado).

O PGRSS da Faculdade de Odontologia não traz um plano de ação para situações de emergência. Porém, a Comissão de Biossegurança da unidade disponibiliza o Manual de Normas de Biossegurança da Faculdade de Odontologia, onde constam as regras de higiene e biossegurança obrigatórias em todas as clínicas e laboratórios. Apesar de relatar as capacitações já realizadas pela equipe envolvida no PGRSS, o plano não apresenta as capacitações a serem desenvolvidas, nem indica inclusão do tema dos RSS na grade curricular do curso de Odontologia ou na forma de educação continuada. Schneider et al. (2015) apontam a formação profissional como aspecto de fundamental importância para o sucesso dos sistemas de gerenciamento de resíduos. Os autores ainda destacam que os profissionais da área da saúde normalmente 
estão empenhados em inúmeras tarefas, podendo não reservar tempo para aprender a manusear os resíduos que geram, o que dificulta o estabelecimento de novos conceitos e comportamentos relacionados aos RSS.

Neste contexto, é fundamental que o tema dos RSS assume seu espaço no currículo dos profissionais que irão atuar na área da saúde, assim como nas propostas de formação continuada dos profissionais já atuantes (SCHNEIDER et al., 2015). A presença de um PGRSS abrangente é necessária para promover a conscientização dos profissionais da área odontológica quanto ao tema dos RSS, sendo que a educação dos futuros dentistas, com o objetivo de melhorar seus conhecimentos sobre o assunto do gerenciamento de resíduos, deve ser considerada sempre que possível (KOOLIVAND et al., 2015).

Os indicadores utilizados para avaliação do PGRSS da Faculdade de Odontologia são: número de atendimentos realizados, variação da geração de resíduos classe I, taxa de acidentes com resíduos perfurocortantes, número de encontros e/ou capacitações ofertadas e frequência da prestação dos serviços de coleta e transporte de RSS. Com base nisso, foi possível verificar que nem todos os indicadores considerados indispensáveis pelos órgãos especializados foram contemplados (BRASIL, 2006). O acompanhamento operacional das ações propostas no PGRSS fica à cargo da Comissão Gestora do plano.

A Comissão Gestora do PGRSS da Faculdade de Odontologia também é responsável pelo monitoramento e avaliação do plano, bem como quaisquer adequações necessários durante sua implantação, considerando o período de vigência do plano de 2 anos. Como a implantação do PGRSS não completou seu período de vigência, não foram realizadas avaliações e revisões do plano até o momento deste trabalho (Tabela 9).

Tabela 9: Verificação das ações realizadas da etapa 7 no PGRSS da Faculdade de Odontologia (FO).

\begin{tabular}{|c|c|}
\hline Etapas para elaboração do PGRSS (BRASIL, 2006) & Análise documental do PGRSS da FO \\
\hline \multicolumn{2}{|l|}{ Avaliação do PGRSS } \\
\hline $\begin{array}{l}\text { - Verificar se os resultados esperados foram ou serão atingidos, } \\
\text { através de quadro de acompanhamento, propondo adaptações ao } \\
\text { plano, se necessário }\end{array}$ & $x$ \\
\hline
\end{tabular}

Legenda: $\checkmark$ (realizado); $X$ (não realizado).

Em pesquisa realizada por Hidalgo (2012), das 50 unidades odontológicas do serviço público estudadas, nenhuma apresentava cópia do PGRSS e o percentual de estabelecimentos que realizava a correta segregação dos resíduos no momento da sua geração foi de $62 \%$. A autora também comenta que em nenhuma das unidades de atendimento odontológico os funcionários utilizavam paramentação completa para a coleta e transporte dos RSS, sendo que apenas $4 \%$ dos estabelecimentos apresentavam equipamento para transporte interno dos resíduos. Na etapa inicial do gerenciamento dos RSS observou-se que os resíduos comuns (invólucros, embalagens e papéis) eram colocados junto aos resíduos contaminados, aumentando o volume de resíduos infecciosos e, consequentemente, os custos ao serviço público para a sua eliminação. Nesse âmbito, ressalta-se que o desenvolvimento de práticas de gerenciamento de RSS, a cuidadosa segregação de resíduos e os programas de treinamento/capacitação, bem como a atenção aos materiais adquiridos, são essenciais para a minimização dos impactos ambientais e à saúde pública de qualquer tecnologia ou atividade (GAUTAM et al., 2010). 
A gestão deve integrar ações relacionadas à tomada de decisões na perspectiva administrativa, operacional, financeira, social e ambiental e têm no planejamento integrado uma importante ferramenta de gerenciamento de resíduos em todas as suas etapas, viabilizando o estabelecimento, de maneira sistemática e integrada, de metas, programas, sistemas organizacionais e tecnologias compatíveis com a realidade local (FERREIRA et al., 2009). O gerenciamento é tido como um processo capaz de minimizar ou até mesmo impedir os efeitos adversos causados pelos RSS, do ponto de vista sanitário, ambiental e ocupacional, sempre que realizado racional e adequadamente (VARAGO et al., 2016).

No que diz respeito à prevenção da poluição ambiental em consultórios odontológicos, estratégias de controle, como substituição de produtos e treinamento de funcionários, devem ser implementadas para reduzir a geração de resíduos e minimizar o efeito potencial de danos na segurança dos funcionários e do meio ambiente (SUSHMA et al., 2012). Programas de minimização (redução), segregação (separação), reuso e reciclagem de resíduos precisam ser implementados sempre que possível para obtenção do melhor gerenciamento de resíduos de serviços de saúde odontológica (NABIZADEH et al., 2012).

\section{CONCLUSÕES}

A partir deste trabalho foi possível verificar que a realização de um diagnóstico das condições de manejo dos resíduos de serviços de saúde, previamente à elaboração do plano de gerenciamento é essencial à sua eficácia nas unidades geradoras deste tipo de resíduo. Embora constatada a importância do diagnóstico de gerenciamento de RSS, o PGRSS da Faculdade de Odontologia foi elaborado sem a realização desta etapa, o que dificulta a elaboração de um plano que considere as particularidades da unidade geradora, principalmente por tratar-se também de uma instituição de ensino superior. Em suma, constatou-se que apenas a existência de um documento físico não garante o correto manejo dos resíduos de serviços de saúde na prática.

Em vista de que as principais falhas observadas no gerenciamento de RSS da Faculdade de Odontologia foram em relação à etapa de segregação dos resíduos, uma vez que foram observadas falhas e omissões de fácil resolução, mas que a sua não conformidade acarreta em altos custos sociais, ambientais e econômicos. Sendo assim, recomenda-se que a gestão de RSS seja parte integrante da formação continuada de professores, técnicos e demais funcionários da faculdade, de forma integrada nas atividades de ensino, pesquisa e extensão, de modo que esse tema seja incluído na rotina da comunidade acadêmica, inclusive como parte da formação acadêmica dos futuros profissionais. Afinal, os profissionais da área da saúde também são responsáveis pelos resíduos por eles gerados.

\section{REFERÊNCIAS}

ALVES, S. C.; GONÇALVES, F. M.; MONTEROSSO, E. P.; GODECKE, M. V.. Gerenciamento de Resíduos de Saúde: estudo de caso em estabelecimentos públicos municipais de Pelotas/RS. Revista Eletrônica em Gestão, Educação e Tecnologia Ambiental. Santa Maria, v.20, n.1, p.105-114, 2016. DOI: http://dx.doi.org/10.5902/2236117019957
AMARANTE, M. G. R.; SILVA, I. C. R.. Gerenciamento dos Resíduos Sólidos Odontológicos: Legislação versus Realidade. In: MOSTRA DE PRODUÇÃO CIENTÍFICA DA PÓSGRADUAÇÃO LATU SENSU DA PUC-GOIÁS, 8. Anais. Goiânia, 2013. 
BAZRAFSHAN, E.; MOHAMMADI, L.; MOSTAFAPOUR, F. K.; MOGHADDAM, A. A.. Dental Solid Waste Characterization and Management in Iran: A Case Study of Sistan and Baluchestan Province. Waste Management \& Research, v.32, n.2, p.157-164, 2014. DOI: http://doi.org/10.1177/0734242X13520063

BOS, A. V. D.; IZADPANAH, A.. Building Capacity for Comprehensive Medical Waste Management in Asia. The Urban Environment, v.52, n.10, p.18-20, 2002.

BRASIL. Agência Nacional de Vigilância Sanitária (Anvisa). Resolução RDC 306: Dispõe sobre o regulamento técnico para o gerenciamento de resíduos de serviços de saúde. Brasília, 2004.

BRASIL. Conselho Nacional do Meio Ambiente (Conama). Resolução Conama 358: Dispõe sobre o tratamento e a disposição final dos resíduos dos serviços de saúde e dá outras providências. Brasília, 2005.

BRASIL. Manual de Gerenciamento de Resíduos de Serviços de Saúde. Agência Nacional de Vigilância Sanitária (Anvisa). Brasília: Ministério da Saúde, 2006.

BRASIL. Serviços Odontológicos: Prevenção e Controle de Riscos. Agência Nacional de Vigilância Sanitária (Anvisa). Brasília: Ministério da Saúde, 2006.

BRASIL. Lei Federal 12.305: Institui a Política Nacional de Resíduos Sólidos; altera a Lei 9.605, de 12 de fevereiro de 1998; e dá outras providências. Diário Oficial [da] República Federativa do Brasil, Poder Executivo. Brasília, DF, 2010.

CORRÊA, L. B.. A Educação Ambiental e os Resíduos Sólidos de Serviços de Saúde: a Formação Acadêmica. Dissertação (Mestrado em Educação Ambiental) - Universidade Federal do Rio Grande, Rio Grande, 2005.

DARWISH, R.; AL-KHATIB, I. A.. Evaluation of dental waste management in two cities in Palestine. Eastern Mediterranean Health Journal, v.2, n.2, p.217-222, 2006.

FERREIRA, D. D. M.; GORGES, J.; SILVA, L. E.. Plano de Gerenciamento de resíduos do serviço de saúde: o caso do setor odontológico de uma entidade sindical. Inter Science Place, v.1, n.9, p.1-18, 2009.

GAUTAM, V.; THAPAR, R.; SHARMA, M.. Biomedical Waste Management: Incineration vs. Environmental Safety. Indian Journal of Medical Microbiology, v.28, n.3, p.191-192, 2010 DOI: http://doi.org/10.4103/0255-0857.66465

HIDALGO, L. R. C. Gerenciamento de resíduos odontológicos no serviço público e seu impacto no meio ambiente. Dissertação (Mestrado em Odontologia Preventiva e Social) - Universidade Estadual Paulista, Araçatuba, 2012.

KOOLIVAND, A.; GHOLAMI-BORUJENI, F.; NOURMORADI, H. Investigation on the characteristics and management of dental waste in Urmia, Iran. Journal of Material Cycles and Waste Management, v.17, n.3, p.553-559, 2015. DOI: http://doi.org/10.1007/s10163-014-0278-2

MAXIMIANO, A. C.; ANSELMO, J. L.. Escritório de gerenciamento de projetos: um estudo de caso. Revista de Administração, v.41, n.4, p.394-403, 2006.
MOREIRA, D. A.. O método fenomenológico na pesquisa. São Paulo: Pioneira Thomson, 2002.

MOURA, L. A. A.. Qualidade e Gestão Ambiental. São Paulo: J. Oliveira, 2004.

NABIZADEH, R.; KOOLIVAND, A.; JAFARI, A.J.; YUNESIAN, M.; OMRANI, G.. Composition and production rate of dental solid waste and associated management practices in Hamadan, Iran. Waste Management \& Research, v.30, n.6, p.619-624, 2012. DOI: http://doi.org/10.1177/0734242X11412110

NASROLLAHI, M.; ZAZOULI, M.; BALARAK, D.; GOLROKHI, M. M.. Quantity and Quality Analysis and Management of Dental Solid Waste Produced in Private Office in Urmia. Journal of Dental School, v.33, n.4, p.262-268, 2015.

OLIVEIRA, C. L.. Um apanho teórico-conceitual sobre a pesquisa qualitativa: tipos, técnicas e características. Revista Travessias, v.11, n.3, 2008

OLIVEIRA, M. C.; MOREIRA, A. C. A.. Gerenciamento dos resíduos produzidos em consultórios odontológicos de Salvador, Bahia. Revista de Ciências Médicas e Biológicas, v.11, n.2, p.194-200, 2012. DOI: http://dx.doi.org/10.9771/cmbio.v11i2.6684

SÁ-SILVA, J. R.; ALMEIDA, C. D.; GUIDANI, J. F.. Pesquisa documental: pistas teóricas e metodológicas. Revista Brasileira de História e Ciência, v.1, n.1, p.1-15, 2009.

SCHNEIDER, V. E.; STEDILE, N. L. R.. Resíduos de serviços de saúde: um olhar interdisciplinar sobre o fenômeno. 3 Ed. Caxias do Sul: Educs, 2015.

SCHNEIDER, V. E.; EMMERICH, R. C. P.. Resíduos de serviços de saúde. In: SCHNEIDER, V. E.; STEDILE, N. L. R.. Resíduos de serviços de saúde: um olhar interdisciplinar sobre o fenômeno. 3 ed. Caxias do Sul: Educs, 2015. p.31-40.

SCHNEIDER, V. E.. Classificação e segregação de resíduos de serviços de saúde como determinantes da eficácia do gerenciamento. In: SCHNEIDER, V. E.; STEDILE, N. L. R.. Resíduos de serviços de saúde: um olhar interdisciplinar sobre o fenômeno. 3 ed. Caxias do Sul: Educs, 2015. p.41-56.

SCHNEIDER, V. E.; EMMERICH, R. C. P.; CALDART, V.; ORLADIN, S. M.. Gerenciamento de resíduos de serviços de saúde. In: SCHNEIDER, V. E.; STEDILE, N. L. R.. Resíduos de serviços de saúde: um olhar interdisciplinar sobre o fenômeno. 3 ed. Caxias do Sul: Educs, 2015. p.79-114.

SCHNEIDER, V. E.; FINKLER, R.. O plano de gerenciamento de resíduos de serviços de saúde como ferramenta de planejamento e gestão. In: SCHNEIDER, V. E.; STEDILE, N. L. R.. Resíduos de serviços de saúde: um olhar interdisciplinar sobre o fenômeno. 3 ed. Caxias do Sul: Educs, 2015. p.133148.

SILVEIRA, D. T.; CÓRDOVA, F. P. A pesquisa científica. In GERHARDT, T. E.; SILVEIRA, D. T.. Métodos de pesquisa. Porto Alegre: UFRGS, 2009. p.31-42. 
SISTEMA FIRJAN. Manual de Gerenciamento de Resíduos: Guia de procedimento passo a passo. Rio de Janeiro: GMA, 2006.

STEDILE, N. L. R.; SCHNEIDER, V. E.; DE CONTO, S. M.; HENKES, A. J.; MALDOTTI, A. C. P. S.; PAIZ, J. C.; KAPPES, A. C.. Gerenciamento de resíduos de serviços de saúde em diferentes fontes geradoras: uma questão de saúde individual e coletiva. In: SCHNEIDER, V. E.; STEDILE, N. L. R.. Resíduos de serviços de saúde: um olhar interdisciplinar sobre o fenômeno. 3 ed. Caxias do Sul: Educs, 2015. p.351374.
SUSHMA, R.; NAGANANDINI, S.; NAGABHUSHANA, D.. Issues Impacting Dental Hospital Waste. Indian Journal of Dental Advancements, v.4, n.2, p.814-821, 2012.

TOGNOC, A. M. G.. Gerenciamento de Resíduos de Serviços de Saúde. In: CONGRESSO NACIONAL DE EXCELÊNCIA EM GESTÃO, 11. Anais. Rio de Janeiro, 2015.

VARAGO, C.; ZUCCHERELLI, E.; SALES, F. S.; SCUR, G.. Gerenciamento de resíduos de serviços de saúde: estudo de caso de dois hospitais de São Paulo. In: ENCONTRO NACIONAL DE ENGENHARIA DE PRODUÇÃO, 36. Anais. João Pessoa, 2016.

A CBPC - Companhia Brasileira de Produção Científica (CNPJ: 11.221.422/0001-03) detém os direitos materiais desta publicação. Os direitos referem-se à publicação do trabalho em qualquer parte do mundo, incluindo os direitos às renovaç̃ões, expansões e disseminações da contribuição, bem como outros direitos subsidiários. Todos os trabalhos publicados eletronicamente poderão posteriormente ser publicados em coletâneas impressas sob coordenação da Sustenere Publishing, da Companhia Brasileira de Produção Científica e seus parceiros autorizados. Os (as) autores (as) preservam os direitos autorais, mas não têm permissão para a publicação da contribuição em outro meio, impresso ou digital, em português ou em tradução. 